\title{
Kawasaki Disease - Options for Anti-Platelet Therapy
}

\begin{abstract}
Alam S*
Sindh Government Hospital Korangi, Karachi, Pakistan

*Corresponding author: Alam Shoaib, Pharmacist, Department of Pharmacy Services, Sindh Government Hospital Korangi, Karachi, Pakistan
\end{abstract}

Received: January 12, 2017; Accepted: February 27, 2017; Published: March 01, 2017

\begin{abstract}
Kawasaki disease is characterized by fever, redness, swelling and inflammation at various parts of body (lips, mouth and throat), conjunctivitis as well as swelling of lymphnodes around neck. The widely accepted therapy is high dose IVIG and acetylsalicylic acid. One of most common complication is coronary artery aneurysms which can lead to thrombosis. For the prevention of this cardiac complication which could be fatal, the patient is provided with platelet disaggregation therapy. There are several pharmacotherapeutic options: aspirin, clopidogrel, dipyridamole, prasugrel, abciximab, ticlopidine, cilostazol etc. Some of these drugs have been successfully used but for remaining the window for study is still open.
\end{abstract}

Keywords: Kawasaki disease; Platelets; Aspirin; Clopidogrel; Ticlopidine; Abciximab; Dipyridamole

\section{Abbreviations}

KD: Kawasaki Disease; CAA: Coronary Artery Aneurysm; ASA: Acetyl Salicylic Acid; IVIG: Intravenous Immune Globulin; ADP: Adenosine diphosphate; Mac-1: Macrophage-1 antigen; cAMP: Cyclic Adenosine Monophosphate; PDE: Phosphodiesterase; GI Gastro Intestinal

\section{Introduction}

Kawasaki Disease (KD) was initially depicted by Tomisaku Kawasaki and this pathology has replaced acute rheumatic fever for being underlining cause of acquired heart disease in paediatric population of developed countries [1]. KD has been shown up in several countries around the world. At present, greater than 60 countries in all continents have stated the cases of $\mathrm{KD}$ [2]. KD is an acute, self-limited vasculitis of unidentified reason which has a prominent tendency for the coronary arteries of infants and young children [3]. Even though it is believed that KD is because of infectious agent in an immunocompromised individual, the culprit agent continues to be indefinable. The disease is distinguished by bilateral nonexudative conjunctivitis, cervical lymphadenopathy, alterations in the extremities, lips and oral mucosa erythema, fever and rash. While $15 \%$ to $25 \%$ of cases advance to coronary artery aneurysms or ectasia if disease is not treated in children and may also progress to ischemic heart disease or unexpected death [4].

\section{Cardiac complications of KD}

CAA is frequent and potentially fatal complication of $\mathrm{KD}$. Around $25 \%$ of untreated patients of KD progress towards CAA and few of them step forward to coronary thrombosis, myocardial infarction and death. Arrhythmias, cardiac failure, mitral or aortic regurgitation, myocarditis, pericardial effusion and pericarditis are other complications associated with heart have been reported in KD. Death in KD is due to myocardial infarction which result from thrombosis of aneurysm or stenosis. It has been verified that even after treating aneurysms, patients may have abnormal vascular wall morphology and vascular dysfunction [5-7].

\section{Aim of treatment}

The aim treatment in $\mathrm{KD}$ is to decrease inflammation as well as avoid the progression towards CAA and arterial thrombosis $[4,8-10]$.

\section{Management}

Intravenous Immune Globulin (IVIG) at dose of $2 \mathrm{gm} / \mathrm{kg}$ remains the backbone treatment for $\mathrm{KD}$. Aspirin is also considered as a part of standard treatment since it acts as an anti-inflammatory and antiplatelet agent. The highly recommended dose of aspirin is $80 \mathrm{mg} / \mathrm{kg}$ to $100 \mathrm{mg} / \mathrm{kg}$ per day into 4 divided doses initially, as absorption from intestine is possibly less in early stage of disease. This high dose of aspirin is continued for 48 to 72 hours, once the patient is afebrile and then the subsequent low dose of aspirin is initiated at dose of $3 \mathrm{mg} / \mathrm{kg}$ to $5 \mathrm{mg} / \mathrm{kg}$ per day. In most of the patients aspirin is discontinued after 6 to 8 weeks after onset of disease (if no coronary changes are noticed); this overlaps time period during which there is high incidence of coronary artery aneurysm development [10].

Approximately 85 to $90 \%$ of cases respond rapidly to the first therapy which includes IVIG and high dose aspirin, Conversely, those having continuing or recurrent fever further than 36 hours of initial pharmacotherapy demand more pharmacological strategies. In such cases second dose of $2 \mathrm{~g}$ per $\mathrm{kg}$ of IVIG is administered. Steroids are employed as a replacement to second IVIG course, but the consequences of steroids on coronary artery aneurysms are controversial. Therefore, some clinicians refuse to give steroids unless fever continues after second dose of IVIG. Other pharmacotherapeutic options include: abciximab, cyclophosphamide (cytotoxic agents), infliximab (monoclonal antibody), pentoxifylline, ulinastatin (a human trypsin inhibitor) and plasma exchange. These agents have been utilized for treatment of KD in few patients [1].

\section{Role of Different Anti-Platelet Agents}

\section{Aspirin}

ASA is extensively used medication in $\mathrm{KD}$ because of its antipyretic as well as anti-inflammatory and antiplatelet capabilities. It works by hindering the production of prostaglandins and it is considered as first drug of choice in children with $\mathrm{KD}$ [11]. 
At the beginning aspirin is administered at high dose and following subsequent doses are then reduced for its anti-thrombotic effects. Initial high dose of aspirin ranges from $30 \mathrm{mg} / \mathrm{kg}$ to $100 \mathrm{mg} /$ $\mathrm{kg}$ per day orally daily, until the normalization of inflammatory parameters [12]. As soon as high dose is stopped, low dose of ASA (anti-platelet dose) at $3 \mathrm{mg} / \mathrm{kg}$ to $5 \mathrm{mg} / \mathrm{kg}$ per day is initiated. If any abnormal features are observed in coronary arteries then low dose ASA is maintained indefinitely [13].

\section{High dose Vs low dose aspirin during acute phase of KD}

It has been observed that high anti-inflammatory doses of aspirin (i.e. 80 to $100 \mathrm{mg} / \mathrm{kg} /$ day), instead of low anti-pyretic dose, cut down the time duration of fever in treated patients in contrast to untreated patients. But the ultimate goal of $\mathrm{KD}$ is not only to narrow down the duration of fever but also to stop the development of CAA. There are lots of debates regarding the high-dose of aspirin [11]. High doses of ASA may range from $30 \mathrm{mg} / \mathrm{kg}$ to $100 \mathrm{mg} / \mathrm{kg}$ per day and varies depending upon the countries for instance $30-50 \mathrm{mg} / \mathrm{kg} /$ day of ASA is used in the United Kingdom and $80 \mathrm{mg} / \mathrm{kg}$ to $100 \mathrm{mg} / \mathrm{kg}$ per day of ASA is used in Japan and in the United. These doses are divided into 4 equal doses for ease in administration [12]. The concerns of hepatotoxicity associated with ASA is the reason for the use of moderate dose (30 to $50 \mathrm{mg} / \mathrm{kg} /$ day) in Japan [14].

The duration for initial high dose of ASA varies from institution to institution. Majority of institutions continue high dose ASA for 48 to 72 hours after the termination of the fever, while other experts continue the high dose up to day $14^{\text {th }}$ of the disease. Few clinicians suggest the use for more than 48 to 72 hours after the patient is afebrile [4]. High doses of ASA may result in gastritis so it is recommended to administer the dose soon after meals [13]. Other undesirable effects associated with prolong use of high dose of ASA are anemia and gastrointestinal bleeding. The anemia associated with high dose is thought to be, because of higher hepcidin level after IVIG treatment. Elevated hepcidin levels cause the reduction in the level of serum iron as well as lower downs the accessibility of iron for erythropoiesis [14].

The results from a retrospective study comprising of 851 patients of KD from two different medical centers, conclude that the high dose of ASA during acute phase of illness do not possess any benefit with respect to inflammation and treatment outcome. Therefore, there is no need to use specifically high doses of aspirin during acute stage [14]. Similarly, a meta-analysis reported no significant change in the development of CAA among the groups which were treated with high dose (i.e. $80 \mathrm{mg} / \mathrm{kg}$ to $120 \mathrm{mg} / \mathrm{kg}$ per day) of ASA and others treated with low dose (i.e. $30 \mathrm{mg} / \mathrm{kg}$ to $50 \mathrm{mg} / \mathrm{kg}$ per day) of ASA. It has been noticed that the dose $30 \mathrm{mg} / \mathrm{kg}$ to $50 \mathrm{mg} / \mathrm{kg}$ per day have better gastrointestinal tolerance with less undesirable effects than the higher doses [15]. It is also observed that Asians are more prone to develop ASA intoxication at high doses therefore the dose of 30 to $50 \mathrm{mg} / \mathrm{kg}$ per day is used widely in Asia [16].

\section{Low dose aspirin during subacute phase of KD}

Low-dose at 3 to $5 \mathrm{mg} / \mathrm{kg}$ per day of ASA during subacute stage of disease is for achieving the platelet disaggregating effect which subsequently reduces the development of thrombosis in coronary arteries [12]. Low dose is maintained for the period of two months after the onset of KD until there is no indication of coronary artery abnormalities on echocardiogram. It should be continued for unspecified time in those who have progressed to coronary artery abnormalities [13].

\section{Long term ASA therapy considerations}

Ibuprofen should not be prescribed in those patients who have been using ASA as it affects the platelet disaggregation induced by ASA. Reye syndrome has been reported in children suffering from KD who have been treated on ASA and hence ASA should be stopped on revelation of either influenza or varicella [13]. In patients who need long-term therapy with aspirin the annual vaccination against influenza virus is recommended to prevent Reye syndrome [12]. Measles vaccine could be postponed for 11 months after the administration of IVIG but risk for delaying of vaccine must be considered against the benefits of earlier vaccination in children who are expected to suffer from measles. In the United States, measles is not that common so the delay is mostly acceptable [17]. Some physicians switch aspirin with other antiplatelet medications for period of six weeks. Parents of the children receiving salicylates should be counselled to contact physician quickly if the child develops symptoms of or is exposed to either influenza or varicella [4]. Gastrointestinal haemorrhage associated with salicylate therapy appears to be infrequent side effect in patients with KD [18]. However, stress ulcer prophylaxis should be included in therapy of those who take high-dose aspirin for prolong duration [4]. There is possibility regarding aspirin associated hemolytic effect in glucose-6-phosphate dehydrogenase (G6PD) deficient individuals. IVIG alone could be better pharmacotherapeutic option in such patients. Moreover, anemia is a common in $\mathrm{KD}$ and it is also related to sustained fever duration [14]. Limited absorption and enhanced clearance have been noticed in children with acute $\mathrm{KD}$, therefore, it is not mandatory to determine ASA levels unless toxicity is suspected [16]. Patients should be monitored for acute symptoms of salicylate toxicity like nausea, vomiting, tinnitus, and hyperventilation as well as delayed symptoms such as hyperactivity, fever, confusion, and seizures [4].

\section{Clopidogrel}

ASA alone is not always enough to stop the progression towards the development of aterial thrombosis as it only interferes single pathway i.e. inhibition of cyclooxygenase [19]. Clopidogrel alters platelet activation by irreversibly impeding adenosine 5 '-diphosphate. Drop off in the concentration of ADP lower downs the activation of the glycoprotein IIb/IIIa complex which is essential for platelet to produce thrombus [20]. Depending upon the severity of coronary artery involvement, other anti-platelet drugs are also considered [12]. Similarly, it has been documented that concomitant use of ASA along with clopidogrel is more efficacious for prevention of thrombosis in both coronary artery and cerebral regions. Few clinicians suggest the combination of ASA and clopidogrel in complicated patients like those with multiple or complex aneurysms [4]. Clopidogrel is choice of anti-platelet agent if ASA is contraindicated [13] and some experts initiate dual anti-platelet therapy, if platelet counts are exceptionally high [10]. Clopidogrel may be considered at a dose of $1 \mathrm{mg} / \mathrm{kg}$ per day up to a maximum dose of $75 \mathrm{mg} /$ day in children allergic to aspirin or concomitant administration of varicella and influenza vaccine along with aspirin [9]. Clopidogrel has emerged as an alternative inhibitor of platelet aggregation with similar efficacy to aspirin and an extremely low risk of myelotoxicity [21]. 
In the case of clopidogrel, a substantial number of the patients are non-responders due to lack of cytochrome P450 2C19 [22]. The * 2 and * 3 alleles of CYP2C19 has been found to decrease antiplatelet effect of clopidogrel because of decrease in production of clopidogrel 's active metabolite. And the prevalence of the * 2 and ${ }^{*} 3$ alleles depends upon the ethnicity for example in Caucasians, Blacks, and Asians, the proportion of patients who carry at least one copy of ${ }^{*} 2$ is $25 \%, 30 \%$, and $40-50 \%$ respectively, while prevalence of 3 is $<1 \%,<1 \%$, and $7 \%$, respectively $[23,24]$. Therefore, it is important to determine reduced cytochrome P450 2C19 individuals if clopidogrel is considered.

\section{Ticlopidine}

Ticlopidine is another antiplatelet agent which has been used in substitution or along with aspirin. It is commonly used in Japan as compared to United States. It works by partial inhibition of Adenosine 59-Diphosphate (ADP) which lower downs the ability of ADP to bring about the alteration in glycoprotein IIb/IIIa complexes necessary for platelet aggregation. Severe neutropenia (1\% to $2 \%$ of patients) associated with ticlopidine, which is not reversible always after termination of drug, is reason behind the limited use of this drug in United States. A case is reported in which ticlopidine have been successfully used along with aspirin in a 7 months old child suffering from KD along with thrombus in a giant coronary aneurysm that failed to resolve with thrombolytic therapy [21].

Another adverse effect associated with ticlopidine is thrombotic thrombocytopenic purpura, therefore, it has been replaced by clopidogrel. Clinicians and patients must remain attentive, as this effect may possibly arise soon after the start of drug and all the cases should be conveyed to Med Watch, FDA's safety information and adverse event reporting program [25].

\section{Abciximab}

Agents which carry out vascular remodelling are the topic of interest for many researchers. Abciximab is a monoclonal antibody which interacts with glycoprotein IIb/IIIa receptor of platelets so as to hinder the aggregation. Agents which produce their antiplatelet response through this pathway have already been used to treat thrombosis in KD efficaciously. Beside this abciximab, pharmacologically possess some more features which are advantageous in KD. Firstly, it attaches to vitronectin receptors and block adhesion of vascular smooth muscle cells to the receptor ligands at the place of vascular inflammation. Furthermore, abciximab may also interact with Mac-1 receptor which interferes circulating monocytes to positions of vascular damage. Abciximab also contributes the remodelling by hindering migration as well as by encouraging apoptosis of smooth muscle cells. It is administered intravenously as a loading bolus dose of $0.25 \mathrm{mg} / \mathrm{kg}$, followed by an infusion of $0.12 \mu \mathrm{g} / \mathrm{kg} /$ minute for 12 hours [26].

In a retrospective study, 9 subjects with CAA were treated with standard therapy for $\mathrm{KD}$ and 6 subjects with similar lesions were treated with standard therapy plus abciximab. The patients which were administered abciximab were superior because of higher rate of resolution of their aneurysms contrast to those who were provided with standard therapy alone [27]. Abciximab emerges to be advantageous in KD patients, particularly in those subjects who progress towards aneurysms [28].

\section{Prasugrel}

Prasugrel irreversibly blocks the P2Y12 ADP receptor on the platelet surface and drop off the aggregation of platelets. It is a prodrug which is rapidly metabolized to an active metabolite with half-life of nearly 4 hours. The cost of therapy is same for both clopidogrel and prasugrel but prasugrel exhibits more potent and rapid inhibition of platelet aggregation as well as there is less inter-subject response variability as compared to clopidogrel [29].

\section{Cilostazol}

Cilostazol is also an antiplatelet agent which is administered orally and its designated use is treatment of Intermittent Claudication (IC) as well as in patients with coronary artery disease [30]. Cilostazol, a cAMP phosphodiesterase inhibitor, also possess vasodilatory properties [31]. Cilostazol blocks uptake of adenosine and enhances extracellular adenosine concentrations which in turn results in vasodilation and anti-platelet properties, at the same time as it attenuates the cardio-tonic effects stimulated by blocking of PDE. It also brings about positive effects on lipid profile [32]. Cilostazol is administered orally at dose of $100 \mathrm{mg}$ twice a day before meals or 2 hours after meal [33]. Cilostazol have never been employed or studied in patients of KD for its antiplatelet and vasodilatory effects in coronary arteries and it is also associated with serious adverse effects like congestive heart failure, gastrointestinal hemorrhage, atrial fibrillation, diarrhea and dyspnea [34].

\section{Dipyridamole}

More than 50 years has been passed since the first time when dipyridamole was synthesized and at the beginning it was employed as a coronary artery vasodilator. Soon it was discovered that dipyridamole inhibits platelet aggregation and stop the development of thrombus in rabbits. The dose of dipyridamole is $200 \mathrm{mg} /$ day for adults [35]. Dipyridamole works by blocking phosphodiesterase and impedes the uptake of adenosine so as to increase platelet cAMP, which in turn potentiates PGI2 and hinders the aggregation. Dipyridamole has been used as an antiplatelet agent in doses of $2-5 \mathrm{mg} / \mathrm{kg} / \mathrm{day}$ is pediatric population [36]. A study has concluded that dipyridamole rises the blood flow in coronary arteries without dilating the proximal aneurysm in paediatrics with a history of $\mathrm{KD}$ [37].

\section{Conclusion}

There are numerous pharmacotherapeutic options regarding the anti-platelet therapy which is proved to be essential. The moderate dose of aspirin initially has similar outcomes as compare to high dose as well as the moderate dose is more convenient in context of gastrointestinal tolerance. Clopidogrel alone and in combination of aspirin have demonstrated promising results but it is necessary to determine whether the individual possess reduced cytochrome P450 2C19 enzymes which require the high doses for antiplatelet effect. Treatment with abciximab have also revealed encouraging outcomes but still there is need of prospective studies to provide solid evidence regarding its efficacy and safety profile. Dipyridamole could be considered in patients with $\mathrm{KD}$ because of its positive effect on coronary blood flow. The use of ticlopidine is limited because of severe adverse effects while for prasugrel and cilostazol there are no studies for its antiplatelet effect on patients of KD. 


\section{References}

1. Freeman AF, Shulman ST. Kawasaki disease: summary of the American Heart Association guidelines. Am Fam Physician. 2006; 74:1141-1148.

2. Uehara R, Belay ED. Epidemiology of kawasaki disease in Asia, Europe, and the United States. Journal of Epidemiology. 2012; 22: 79-85.

3. Fimbres AM, Shulman ST. Kawasaki disease. Pediatrics in Review. 2008; 29: 308 .

4. Newburger JW, Takahashi M, Gerber MA, Gewitz MH, Tani LY, Burns JC, et al. Diagnosis, treatment, and long-term management of Kawasaki disease a statement for health professionals from the Committee on Rheumatic Fever, Endocarditis and Kawasaki Disease, Council on Cardiovascular Disease in the Young, American Heart Association. Circulation. 2004; 110: 2747-2771.

5. Maconochie IK. Kawasaki disease. Archives of disease in childhoodEducation \& practice edition. 2004; 89: 3-8.

6. Tizard EJ. Complications of Kawasaki disease. Current Paediatrics. 2005; 15: 62-68.

7. Aldemir-Kocabaş B, Karbuz A, Karadeniz C, Çiftçi Ö, Özdemir H, Bolkent MG, Uçar T, Tutar E, Atalay S, Fitöz S, Ciftçi E. Another face of Kawasak disease. Turk J Pediatr. 2014; 56: 392-398.

8. Brogan PA, Bose A, Burgner D, Shingadia D, Tulloh R, Michie C, Klein N Booy R, Levin M, Dillon MJ. Kawasaki disease: an evidence based approach to diagnosis, treatment, and proposals for future research. Archives of disease in childhood. 2002; 86: 286-290.

9. Falcini F, Capannini S, Rigante D. Kawasaki syndrome: an intriguing disease with numerous unsolved dilemmas. Pediatric rheumatology. 2011; 9: 1.

10. Satou GM, Giamelli J, Gewitz MH. Kawasaki disease: diagnosis, management, and long-term implications. Cardiology in review. 2007; 15: 163-169.

11. Ichida F, Fatica NS, Engle MA, O'Loughlin JE, Klein AA, Snyder MS, Ehlers $\mathrm{KH}$, Levin AR. Coronary artery involvement in Kawasaki syndrome in Manhattan, New York: risk factors and role of aspirin. Pediatrics. 1987; 80: 828-835.

12. De Rosa GA, Pardeo M, Rigante D. Current recommendations for the pharmacologic therapy in Kawasaki syndrome and management of its cardiovascular complications. Eur Rev Med Pharmacol Sci. 2007; 11: 301 308.

13. Gupta-Malhotra M, Rao PS. Current perspectives on Kawasaki disease. The Indian Journal of Pediatrics. 2005; 72: 621-629.

14. Kuo HC, Lo MH, Hsieh KS, Guo MM, Huang YH. High-dose aspirin is associated with anemia and does not confer benefit to disease outcomes in kawasaki disease. PloS one. 2015; 10: 0144603.

15. Eleftheriou D, Levin M, Shingadia D, Tulloh R, Klein NJ, Brogan PA. Management of Kawasaki disease. Archives of disease in childhood. 2014 99: 74-83.

16. Rowley AH, Shulman ST. Pathogenesis and management of Kawasaki disease. Expert review of anti-infective therapy. 2010; 8: 197-203.

17. Gerding R. Kawasaki disease: a review. Journal of Pediatric Health Care. 2011; 25: 379-387

18. Matsubara T, Mason W, Kashani IA, Kligerman M, Burns JC. Gastrointestinal hemorrhage complicating aspirin therapy in acute Kawasaki disease. The Journal of pediatrics. 1996; 128: 701-703.

19. Li JS, Yow E, Berezny KY, Bokesch PM, Takahashi M, Graham TP, Sanders SP, Sidi D, Bonnet D, Ewert P, Jennings LK. Dosing of Clopidogrel for Platelet Inhibition in Infants and Young Children Primary Results of the Platelet Inhibition in Children On cLOpidogrel (PICOLO) Trial. Circulation. 2008; 117: 553-559.
20. Johnson PN, Kuhn RJ. Combination thrombolytic and anti-platelet therapies in an infant with incomplete Kawasaki disease and coronary aneurysms. The Journal of Pediatric Pharmacology and Therapeutics. 2008; 13: 242-250.

21. O'Brien M, Parness IA, Neufeld EJ, Baker AL, Sundel RP, Newburger JW. Ticlopidine plus aspirin for coronary thrombosis in Kawasaki disease. Pediatrics. 2000; 105

22. Zetterberg F, Svensson P. State of affairs: Design and structure-activity relationships of reversible $\mathrm{P} 2 \mathrm{Y} 12$ receptor antagonists. Bioorganic \& medicinal chemistry letters. 2016; 26: 2739-2754.

23. Mega JL, Close SL, Wiviott SD, Shen L, Hockett RD, Brandt JT, Walker JR, Antman EM, Macias W, Braunwald E, Sabatine MS. Cytochrome p-450 polymorphisms and response to clopidogrel. New England Journal of Medicine. 2009; 360: 354-362.

24. Frere C, Cuisset T, Gaborit B, ALESSI MC, HULOT JS. The CYP2C19* 17 allele is associated with better platelet response to clopidogrel in patients admitted for non-ST acute coronary syndrome. Journal of Thrombosis and Haemostasis. 2009; 7: 1409-1411.

25. Jacob S, Dunn BL, Qureshi ZP, Bandarenko N, Kwaan HC, Pandey DK et al. Ticlopidine-, clopidogrel-, and prasugrel-associated thrombotic thrombocytopenic purpura: a 20-year review from the Southern Network on Adverse Reactions (SONAR). InSeminars in thrombosis and hemostasis. Thieme Medical Publishers. 2012; 38: 845-853.

26. McCandless RT, Minich LL, Tani LY, Williams RV. Does abciximab promote coronary artery remodeling in patients with Kawasaki disease?. The American journal of cardiology. 2010; 105: 1625-1628.

27. Seaton KK, Kharbanda A. Evidence-based management of Kawasaki disease in the emergency department. Pediatric emergency medicine practice. 2015 12: $1-20$.

28. Kuo HC, Chang WC. Cardiovascular Lesions of Kawasaki Disease: From Genetic Study to Clinical Management. INTECH Open Access Publisher; 2012

29. Akbari SH, Reynolds MR, Kadkhodayan Y, Cross DT, Moran CJ. Hemorrhagic complications after prasugrel (Effient) therapy for vascular neurointerventional procedures. Journal of neurointerventional surgery. 2012 May 3: neurintsurg-2012.

30. Soga Y, Yokoi H, Kawasaki T, Nakashima H, Tsurugida M, Hikichi Y, Nobuyoshi M. Efficacy of cilostazol after endovascular therapy for femoropopliteal artery disease in patients with intermittent claudication. Journal of the American College of Cardiology. 2009; 53: 48-53.

31. Yoon YS, Shim WH, Lee DH, Pyun WB, Kim IJ, Jang Y, Cho SY. Usefulness of cilostazol versus ticlopidine in coronary artery stenting. The American journal of cardiology. 1999; 84: 1375-1380.

32. Liu Y, Shakur Y, Yoshitake M, Kambayashi JI. Cilostazol (pletal ${ }^{\circledR}$ ): A dua inhibitor of cyclic nucleotide phosphodiesterase type 3 and adenosine uptake. Cardiovascular drug reviews. 2001; 19: 369-386.

33. Medscape, Drugs and Diseases, Cilostazol. 2015.

34. Chi YW, Lavie CJ, Milani RV, White CJ. Safety and efficacy of cilostazol in the management of intermittent claudication. Vasc Health Risk Manag. 2008; 4: 1197-1203.

35. Gresele P, Momi S, Falcinelli E. Anti-platelet therapy: phosphodiesterase inhibitors. British journal of clinical pharmacology. 2011; 72: 634-646.

36. Mohanty S, Vaidyanathan B. Anti-platelet agents in pediatric cardiac practice Annals of pediatric cardiology. 2013; 6: 59.

37. Kobayashi T, Sone K. Effect of dipyridamole on the blood flow in coronary aneurysms resulting from Kawasaki disease. Pediatric cardiology. 1994; 15 : 263-267.
Austin J Pharmacol Ther - Volume 5 Issue 1 - 2017 ISSN: 2373-6208 | www.austinpublishinggroup.com Alam. (C) All rights are reserved
Citation: Alam S. Kawasaki Disease - Options for Anti-Platelet Therapy. Austin J Pharmacol Ther. 2017; 5(1).1089. 